Research Paper

\title{
Utility of Preoperative Inflammatory Markers to Distinguish Epithelial Ovarian Cancer from Benign Ovarian Masses
}

\author{
Lian $\mathrm{Li}^{1 *}$, Jing Tian ${ }^{1,2 *}$, Liwen Zhang1*, Luyang Liu ${ }^{1}$, Chao Sheng ${ }^{1}$, Yubei Huang1, Hong Zheng1, Fengju \\ Song ${ }^{1}$, Kexin Chen ${ }^{1 凶}$
}

1. Department of Epidemiology and Biostatistics, National Clinical Research Center for Cancer, Key Laboratory of Cancer Prevention and Therapy of Tianjin, Tianjin Medical University Cancer Institute and Hospital, Tianjin, China.

2. Department of Urology, National Clinical Research Center for Cancer, Key Laboratory of Cancer Prevention and Therapy of Tianjin, Tianjin Medical University Cancer Institute and Hospital, Tianjin, China.

* These authors contributed equally to this work.

$\triangle$ Corresponding author: Kexin Chen, M.D., Ph.D., Professor and Chair, Department of Epidemiology and Biostatistics, National Clinical Research Center for Cancer, Key Laboratory of Cancer Prevention and Therapy of Tianjin, Tianjin Medical University Cancer Institute and Hospital, Huanhu Xi Road, Tiyuan Bei, Hexi District, Tianjin, P.R. China 300060. Tel.: ++86 (0)2223372231; Fax: ++86 (0)2223372231; E-mail: chenkexin@timuch.com.

() The author(s). This is an open access article distributed under the terms of the Creative Commons Attribution License (https://creativecommons.org/licenses/by/4.0/). See http:/ /ivyspring.com/terms for full terms and conditions.

Received: 2020.08.06; Accepted: 2021.02.13; Published: 2021.03.05

\begin{abstract}
Background: Inflammatory markers have been reported to be predictors for the presence of epithelial ovarian cancer (EOC), however, the cut-off value of each marker remains unclear and predictive capability of the markers in different histology types of EOC is still unknown.

Methods: A total of 207 patients with benign ovarian masses and 887 EOC patients who underwent surgical resection, and were pathologically diagnosed were included. We compared the difference of preoperative inflammatory markers between benign ovarian masses and EOC patients. Stratified analysis by histology subtype was further conducted. Logistic regression analyses and receiver operating characteristic (ROC) curves was used to evaluate the predictive capability of the markers.

Results: Neutrophil-to-lymphocyte ratio (NLR), platelet-to-lymphocyte ratio (PLR), and lymphocyte-to-monocyte ratio (LMR) were significantly associated with all stages and subtypes of EOC $(P<0.001)$. The optimal cut-off points based on ROC curve analyses for NLR, PLR, and LMR were found

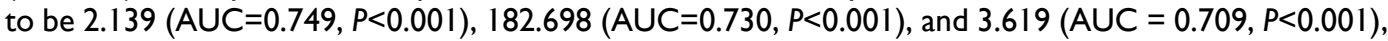
respectively. In low CA125 level patients, high level of NLR and PLR increase the risk of endometrioid EOC, while low level of LMR were significantly associated with an increased risk of serous EOC.

Conclusions: In addition to CA125, NLR, PLR, and LMR could be used as predictors of EOC and preoperative inflammatory markers may be used as a potential biomarker for predicting different histotypes of EOC.
\end{abstract}

Key words: Epithelial ovarian cancer, Inflammation biomarkers, Diagnosis, Benign ovarian masses, Cancer biomarkers.

\section{Introduction}

Epithelial ovarian cancer (EOC) is a major type of ovarian cancer and is the leading cause of death in patients with gynecologic malignancies [1]. Among adnexal masses, nearly $75 \%$ are benign, $15 \%$ are EOC, and $10 \%$ are borderline or other types of malignant ovarian cancers $[2,3]$. Histological subtypes of EOC including serous, clear cell, endometrioid, and mucinous carcinoma et al. Benign ovarian masses have much better prognosis than EOC and the tumor pathology and differentiation between benign ovarian masses and EOC affect the subsequent treatment decision [4]. However, it is difficult to distinguish 
benign and malignant prior to surgery when ovarian masses are found.

Besides patient's age, clinical symptoms, physical examination, the most widely used clinical tools to distinguish benign and malignant of adnexal masses are imaging modalities and tumor markers. Several algorithms such as RMI [5, 6], risk of ovarian malignancy algorithm (ROMA) [7], and the multivariate index assay (OVA1) [8] have been used to evaluate the malignancy potential of adnexal masses. Cancer antigen 125 (CA125) is the most widely used tumor marker in ovarian cancer [5, 9], However, the sensitivity and specificity of CA125 for the diagnosis of EOC is not ideal and CA125 alone should not be used to distinguish between benign and malignant adnexal mass [10]. Therefore, it is necessary to identify additional biomarkers which combined with CA125 to improve the diagnosis accuracy of presence EOC.

Inflammation has been reported to play important role in carcinogenesis and cancer progression [11]. Several inflammation related markers, including neutrophils to lymphocyte ratios (NLR), platelet to lymphocyte ratios (PLR), and lymphocyte to monocyte ratio (LMR) et al. have been shown to be a diagnosis or prognosis makers of cancers [12-16]. These makers could be acquired from routine blood test, which known to be cost-effective, reproducible, less invasive, and universally accepted test currently. Previous studies have also reported that these inflammatory markers could distinguish benign ovarian masses and ovarian cancers [17-20]. However, the cut-off value remains unclear and the accuracy of diagnosis in different histology types of EOC remains unknown [18, 20, 21]. Therefore, we conducted a large sample size retrospective study to identify new biomarkers to assist CA-125 in distinguishing benign masses and EOC. In this study, we propose the optimal cutoff of NLR, PLR, and LMR to predict the presence of EOC, and evaluate the prediction performance in different histology types of EOC and provide evidence for clinical practice.

\section{Materials and methods}

\section{Populations}

Patients who underwent primary surgery in the department of Gynecological oncology at Tianjin Medical University Cancer Institute and Hospital between 2007 and 2017 were recruited. A total of 1707 patients were included according to following inclusion criteria: (1) underwent primary surgery and histologically confirmed for EOC or benign ovarian masses; (2) conducted blood test preoperative routine blood test (2 weeks before surgery), and CA125 information. Patients were excluded if they (1) underwent radiotherapy, chemotherapy, targeted drugs, or other anti-tumor therapy prior to surgery (280 patients); (2) had a history of autoimmune diseases or other malignancies (152 patients). (3) had borderline ovarian cancer (164 patients). (4) had no demographic information (17 patients). Finally, a total of 1094 patients (207 patients with benign ovarian masses and 887 EOC patients) were included for the analysis. Informed consent was obtained for use of the medical records for research purposes and this study was approved by the institutional review board (IRB) of Tianjin Medical University Cancer Institute and Hospital.

\section{Medical records data}

Demographic data, clinical data, pathology report, and laboratory report were retrospectively obtained from medical records. Body mass index (BMI) refers to weight $(\mathrm{kg})$ divided by the square of height $(\mathrm{m})$. Tumor stage was divided into four stages (I, II, III, and IV) according to International Federation of Gynaecology and Obstetrics (FIGO) 2009 Criteria. Then we further classified into early stage (I and II) and advanced stage (III and IV) groups. Pathological diagnosis was divided into two groups: benign ovarian masses (serous cystadenoma, mucinous cystadenoma, mature teratoma, etc.) and epithelial ovarian cancer (serous ovarian cancer, mucinous ovarian cancer, endometrioid ovarian cancer, and other epithelial ovarian cancer). Tumor diameter was classified into two groups of $<5 \mathrm{~cm}$ and $\geq 5 \mathrm{~cm}$. Optimal cytoreduction was considered as the residual tumor size $\leq 1 \mathrm{~cm}$.

Blood cell counts were obtained from the routine blood test that was conducted within a week before operation. NLR refers to the ratio of the absolute neutrophils count to absolute lymphocyte count. PLR refers to the ratio of the absolute platelet count to the absolute lymphocyte count. LMR refers to the ratio of the absolute lymphocyte count to the absolute monocyte count.

\section{Statistical analysis}

To compare the demographic characteristics and preoperative markers between the benign and malignant groups, Student's t-test was used to analysis the variables with normal distributions, and Mann-Whitney U-test was used to analysis the variables with non-normal distributions. Categorical variables were presented as $\mathrm{n}(\%)$ and analyzed using $X^{2}$ test. Logistic regression analyses were used to evaluate the association between the biomarkers and malignancy risk. The receiver-operating characteristic (ROC) curve analysis was used to assess the 
discriminative role of markers and determine the appropriate cut-off values. Subgroup analysis was performed according to stage, histological subtypes and menopausal status. A two-tailed $P$ value $<0.05$ was considered statistically significant. All statistical analysis was performed using SAS 9.4 and R version 3.4.3.

\section{Results}

\section{Patient characteristics}

This study included 1094 patients in total, 207 patients were benign ovarian masses and 887 of whom were EOC. The baseline characteristics of the patients were shown in Table S1 and Table S2. The significant difference was observed in age and menopause status between benign ovarian masses group and EOC groups $(P<0.001)$. And there was no significant difference between the two groups in other demographic and life style factors including BMI, family history of cancer, smoking, and age of menarche et al. The histological subtypes of most EOC patients presented with serous $(n=477,53.78 \%)$ and endometrioid ( $n=194,21.87 \%)$. Serous cystadenoma and mucinous cystadenoma accounted for 15.94\% $(n=33)$ and $5.31 \%(n=11)$ in terms of benign ovarian masses, respectively.

\section{Comparison of variables between benign ovarian masses and EOC groups}

The median levels of CA125, HGB, WBC, NLR, PLR, and LMR were significantly different between benign masses and EOC groups (All $P<0.001$ ) (Table $1)$. In addition, the significant differences were also observed in these variables in the stratified analysis according to histological subtypes of EOC compared to benign ovarian masses (All $P<0.001$ ) (Table 2). Univariate and multivariate logistic regression model showed that the aforementioned markers were significantly associated with the presence of EOC (Table S3).

\section{ROC curve analyses}

Receiver operating characteristic curves for EOC prediction were presented in Table 3. CA125 performed best for the discrimination of benign from EOC (AUC $=0.906,95 \% \mathrm{CI}$ : 0.885-0.928), followed by NLR (AUC $=0.749, \quad 95 \%$ CI: $0.714-0.784), \quad$ PLR (AUC $=0.730, \quad 95 \% \mathrm{CI}: \quad 0.696-0.764)$, and LMR (AUC $=0.709$, 95\%CI: 0.672-0.745). Moreover, PLR, NLR, and LMR still have good performance in distinguishing benign and malignant ovarian masses after adjusting for age (Table S4) and in stratified analysis by stage, histological subtypes, and menopausal status (Table S5- Table S10, Figure S1Figure S3).

The optimal cut-off points based on ROC curve analyses for NLR, PLR, and LMR were found to be 2.139 (sensitivity 0.676, specificity 0.718), 182.698 (sensitivity 0.511 , specificity 0.865 ) and 3.619 (sensitivity 0.536, specificity 0.830), respectively (Figure 1). For CA125, levels higher than cut-off value (35UI/L) had the $89.5 \%$ sensitivity and $70.9 \%$ specificity for the diagnosis of EOC.

Table 1. Comparison of variables between benign ovarian masses and malignant ovarian masses groups.

\begin{tabular}{llllll}
\hline & \multicolumn{3}{l}{ Benign ovarian masses } & \multicolumn{3}{l}{ Malignant ovarian cancer } & \multirow{2}{*}{$P$ value } \\
\cline { 2 - 5 } & $N$ & Median $(\mathrm{IQR})$ & $N$ & Median $(\mathrm{IQR})$ & \\
\hline CA125 $(\mathrm{U} / \mathrm{mL})$ & 199 & $17.91(36.0)$ & 841 & $606.0(1221.7)$ & $<0.001$ \\
HGB $(\mathrm{g} / \mathrm{L})$ & 207 & $130.0(14.0)$ & 885 & $126.0(17.0)$ & $<0.001$ \\
MCV $(\mathrm{Fl})$ & 207 & $90.5(5.4)$ & 885 & $89.4(5.9)$ & 0.005 \\
HCT $(\%)$ & 207 & $39.3(4.3)$ & 884 & $38.6(4.3)$ & 0.002 \\
WBC $\left(10^{\wedge} 9 / \mathrm{L}\right)$ & 206 & $5.76(2.13)$ & 884 & $6.55(2.29)$ & $<0.001$ \\
NLR & 206 & $1.68(1.01)$ & 883 & $2.61(1.83)$ & $<0.001$ \\
PLR & 207 & $131.0(59.1)$ & 882 & $184.6(122.6)$ & $<0.001$ \\
LMR & 206 & $4.87(2.30)$ & 881 & $3.50(2.58)$ & $<0.001$ \\
\hline
\end{tabular}

CA125, Cancer Antigen 125; HGB, hemoglobin concentration; MCV, mean corpuscular volume; HCT, hematocrit; WBC, white blood cell count; NLR, neutrophil-to-lymphocyte ratio; PLR, platelet-to-lymphocyte ratio; LMR, lymphocyte lymphocyte-to-monocyte ratio; IQR, interquartile range.

Table 2. Comparison of variables between benign ovarian masses and malignant ovarian masses of different pathological types.

\begin{tabular}{|c|c|c|c|c|c|c|c|c|c|c|c|}
\hline & \multicolumn{2}{|c|}{ Benign ovarian masses } & \multicolumn{3}{|c|}{ Serous } & \multicolumn{3}{|c|}{ Endometrioid } & \multicolumn{3}{|c|}{ Others } \\
\hline & $N$ & Median (IQR) & $N$ & Median (IQR) & $P^{*}$ & $N$ & Median (IQR) & $P^{*}$ & $N$ & Median (IQR) & $P^{*}$ \\
\hline CA125 (U/mL) & 199 & $17.91(36.0)$ & 457 & $748.0(1333.3)$ & $<0.001$ & 181 & $568.4(1110.6)$ & $<0.001$ & 203 & $272.6(952.4)$ & $<0.001$ \\
\hline $\operatorname{HGB}(g / L)$ & 207 & $130.0(14.0)$ & 476 & $126.0(17.0)$ & $<0.001$ & 194 & $126.0(16.8)$ & 0.001 & 215 & $126.0(18.0)$ & 0.001 \\
\hline $\mathrm{MCV}(\mathrm{Fl})$ & 207 & $90.5(5.4)$ & 476 & $89.4(5.8)$ & 0.006 & 194 & $89.0(5.6)$ & 0.005 & 215 & $90.0(6.2)$ & 0.196 \\
\hline HCT $(\%)$ & 207 & $39.3(4.3)$ & 475 & $38.5(4.4)$ & 0.002 & 194 & $38.7(4.2)$ & 0.055 & 215 & $38.8(4.3)$ & 0.022 \\
\hline WBC $\left(10^{\wedge} 9 / \mathrm{L}\right)$ & 206 & $5.76(2.13)$ & 475 & $6.48(2.13)$ & $<0.001$ & 194 & $6.79(2.48)$ & $<0.001$ & 215 & $6.52(2.28)$ & $<0.001$ \\
\hline NLR & 206 & $1.68(1.01)$ & 475 & $2.56(1.73)$ & $<0.001$ & 192 & $2.72(1.77)$ & $<0.001$ & 215 & $2.60(2.26)$ & $<0.001$ \\
\hline PLR & 207 & $131.0(59.1)$ & 475 & $187.5(133.0)$ & $<0.001$ & 192 & $180.0(89.3)$ & $<0.001$ & 214 & 180.7 (149.3) & $<0.001$ \\
\hline LMR & 206 & $4.87(2.30)$ & 472 & $3.51(2.29)$ & $<0.001$ & 192 & $3.35(2.32)$ & $<0.001$ & 215 & $3.61(3.04)$ & $<0.001$ \\
\hline
\end{tabular}

CA125, Cancer Antigen 125; HGB, hemoglobin concentration; MCV, mean corpuscular volume; HCT, hematocrit; WBC, white blood cell count; NLR, neutrophil-to-lymphocyte ratio; PLR, platelet-to-lymphocyte ratio; LMR, lymphocyte lymphocyte-to-monocyte ratio; IQR, interquartile range.

${ }^{*} P$ value for each group compared with benign ovarian masses. 

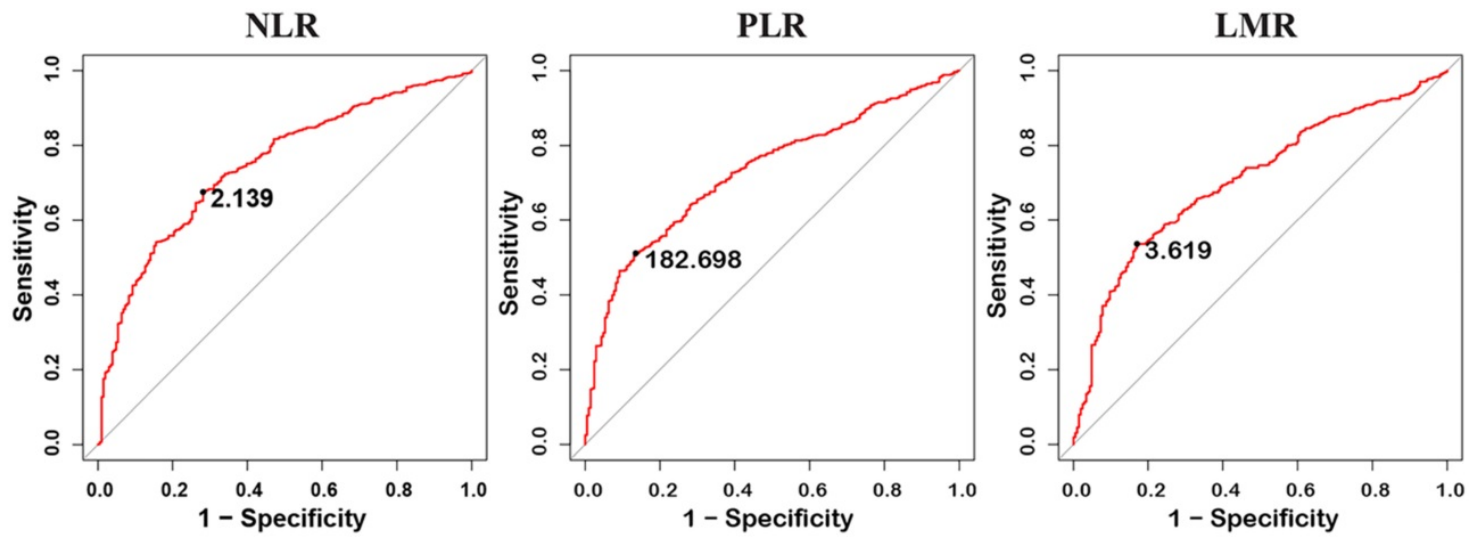

Figure 1. Receiver operating characteristic (ROC) curves analysis of neutrophil-lymphocyte ratio (NLR), platelet-lymphocyte ratio (PLR), and lymphocyte-monocyte ratio (LMR) for discriminating between malignant ovarian cancer and benign ovarian masses.

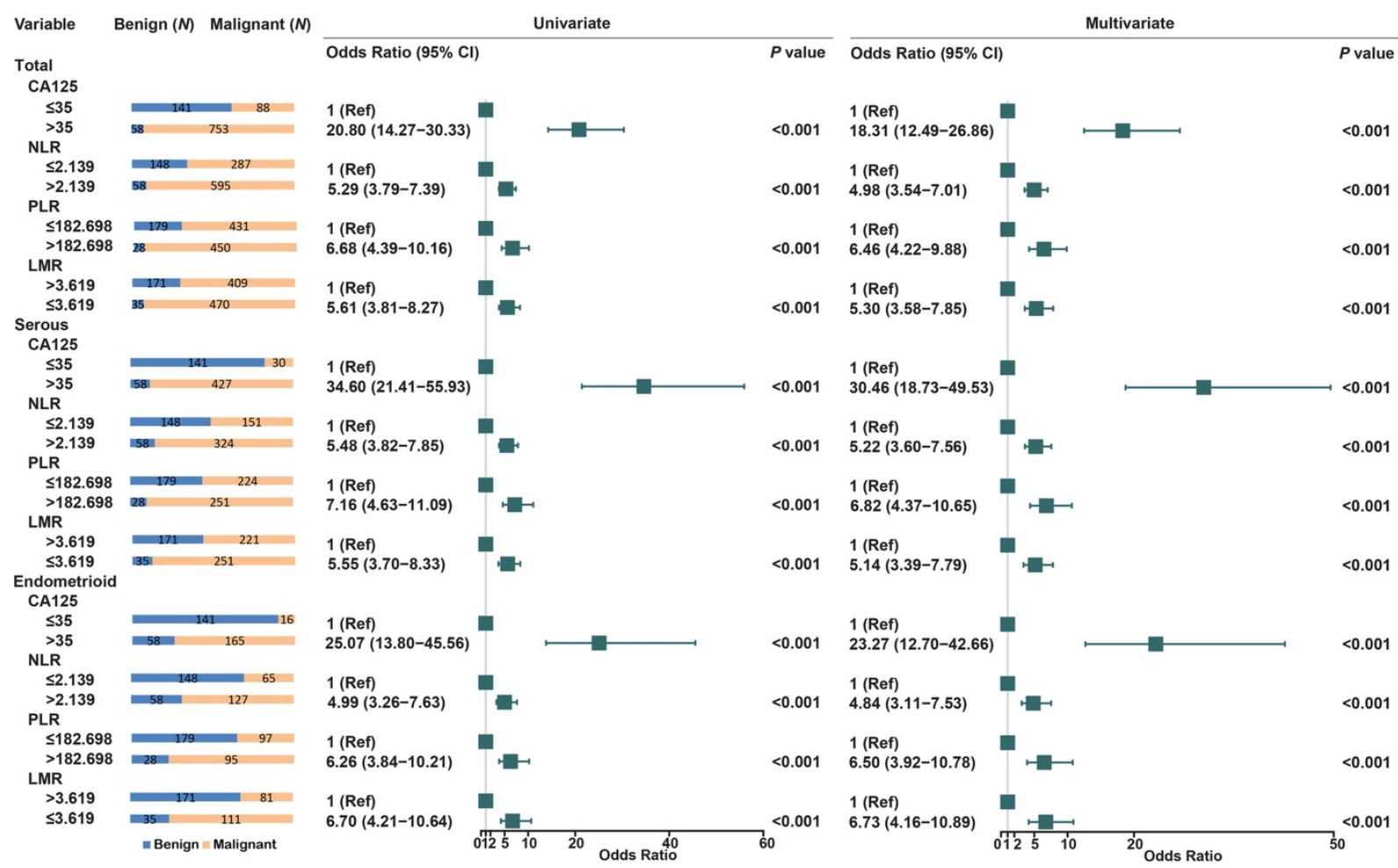

Figure 2. Univariate and multivariate analyses for EOC risk in stratified analysis by histological subtypes.

Table 3. Area under the curve (AUC) for discriminating all malignant cases from benign cases.

\begin{tabular}{llllll}
\hline Variables & AUC & 95\% CI & Cut-off & Sensitivity (\%) & Specificity (\%) \\
\hline CA125 (U/mL) & 90.6 & $88.5-92.8$ & 35 & 89.5 & 70.9 \\
HGB (g/L) & 59.9 & $55.7-64.0$ & 124.5 & 45.1 & 71.0 \\
MCV (Fl) & 56.2 & $51.9-60.6$ & 87.15 & 31.0 & 80.7 \\
HCT (\%) & 56.8 & $52.5-61.0$ & 38.95 & 54.9 & 57.0 \\
WBC (10^9/L) & 63.6 & $59.4-67.8$ & 5.815 & 69.5 & 52.4 \\
NLR & 74.9 & $71.4-78.4$ & 2.139 & 67.6 & 71.8 \\
PLR & 73.0 & $69.6-76.4$ & 182.698 & 51.1 & 86.5 \\
LMR & 70.9 & $67.2-74.5$ & 3.619 & 53.6 & 83.0 \\
CA125, Cancer Antigen 125; HGB, hemoglobin concentration; MCV, mean \\
corpuscular volume; HCT, hematocrit; WBC, white blood cell count; NLR, \\
neutrophil-to-lymphocyte ratio; PLR, platelet-to-lymphocyte ratio; LMR, \\
lymphocyte-to-monocyte ratio.
\end{tabular}

We further assessed the association between these biomarkers and EOC risk (Figure 2). Compared to $\mathrm{CA} 125 \leq 35, \mathrm{CA} 125>35$ have increased the risk of EOC nearly 20-fold (OR $=20.80,95 \% \mathrm{CI}: 14.27-30.33$ ). High levels of NLR (OR $=5.29,95 \%$ CI: 3.79-7.39) and PLR $(\mathrm{OR}=6.68,95 \%$ CI: 4.39-10.16) were also significantly associated with EOC risk. LMR $\leq 3.619$ increase the risk of EOC (OR $=5.61,95 \% \mathrm{CI}$ : 3.81-8.27), when compared to LMR $>3.619$. Histological stratified analyses showed that NLR, PLR and LMR were also increase the risk of serous, endometrioid, and other types of EOC. 


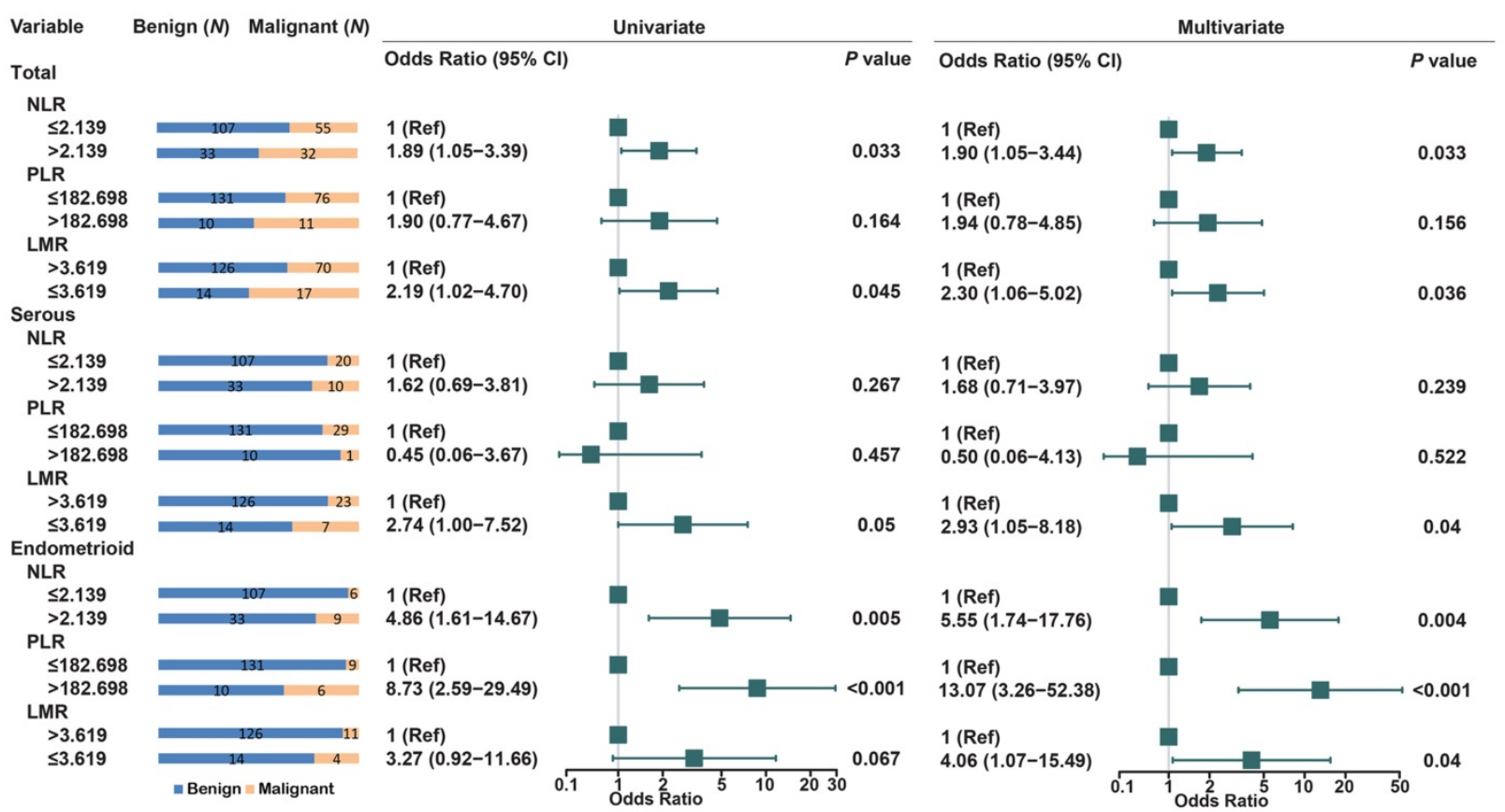

Figure 3. Univariate and multivariate analyses for EOC risk in stratified analysis by histogical subtypes in low level of CA125 patients (CA125 $\leq 35)$.

Of 841 EOC patients, CA125 level was not elevated in 88 EOC patients (CA125 35 ). Therefore, we compared the levels of NLR, PLR, and LMR between 141 benign masses and 88 EOC patients (CA125 $\leq 35)$. Of the three biomarkers, high level of NLR (OR $=1.89,95 \%$ CI: 1.05-3.39) and low level of LMR (OR $=2.19,95 \%$ CI: 1.02-4.70) was significantly associated with the risk of EOC. In stratified analysis by histology, only low level of LMR was significantly increased the risk of serous ovarian cancers $(\mathrm{OR}=$ 2.74, 95\% CI: 1.00-7.52). High level of NLR and PLR increased the risk of endometrioid EOC $(\mathrm{OR}=4.86$, 95\% CI: 1.61-14.67; OR=8.73, 95\% CI: 2.59-29.49) (Figure 3). In addition, NLR, PLR and LMR was associated with all stages and subtypes of EOC in 58 benign ovarian mass and 753 EOC with high CA125 level (CA125>35) (Figure S4- Figure S7).

\section{Discussion}

In current study, we evaluated the utility of preoperative parameters of complete blood count to distinguish EOC from benign ovarian masses and proposed the optimal cutoff of NLR, PLR, and LMR to predict the presence of EOC. In addition, we showed the prediction performance in different pathology types of EOC for the first time.

CA125 is the most commonly used serological marker for ovarian cancer. A number of studies have demonstrated that CA125 can be used for the diagnosis of ovarian cancer $[2,5-8,19,22-27]$, which is consistent with our study. However, nearly $20 \%$ of patients of EOC have lower level of CA125, therefore, CA125 test alone should not be used to differentiate between a benign and a malignant adnexal mass [5, 10]. In the present study, we found that NLR, PLR, and LMR, in combination with CA125 could contribute to predict the presence of EOC.

A number of studies have been reported that NLR, PLR, and LMR could be used as a diagnosis and prognosis marker of ovarian cancer patients $[3,17-20$, 28-33]. Cho et al. [17] found that the preoperative NLR of patients with ovarian cancer was significantly higher than that of patients with benign ovarian masses. The cut-off value based on ROC curve analysis was 2.60 , and the sensitivity and specificity were $66.1 \%$ and $82.7 \%$, respectively. Polat et al. found that the optimal cutoff value of the NLR was 2.47, with $63.4 \%$ sensitivity and $63.5 \%$ specificity for malignancy prediction [30]. Wan et al. reported that preoperative NLR, with a cut-off value of 2.64 , is an independent predictor of EOC [20]. In present study, we reached similar results. The optimal cut-off value based on ROC curve analysis was 2.139 , with a sensitivity of $71.8 \%$ and a specificity of $67.6 \%$ for ovarian cancer prediction. Raungkaewmanee et al. [34] found that PLR of 200 yielded the most optimal predictive value to predict advanced stage disease, and the AUC was 0.66 (95\% CI, 0.59 to 0.73$)$ while the sensitivity and specificity were $59.0 \%$ and $72.7 \%$, respectively. Similar to our results, we evaluated the predictive value of PLR in the diagnosis of ovarian cancer and found a PLR of 182.698 yielded a good predictive value. However, the PLR had an AUC of 0.730 , with the highest specificity among other blood parameters but with relatively lower sensitivity. Previous studies have demonstrated that low level of 
LMR was an independent risk factors for progression-free survival (PFS) and overall survival (OS) in EOC patients, and the cut off values of these studies ranged from 2.07 to 3.84 [31, 35, 36]. Eo et al. [20] identified LMR as a predictor of the presence of EOC in patients with an ovarian mass $(\mathrm{OR}=0.51, \mathrm{P}=$ 0.024). Preoperative LMR with a cut-off value of 3.52 predicted the presence of EOC. In our study, the cut-off value for LMR was 3.619 , by the ROC curve analysis, is an independent predictive factor in EOC patients. We also found that NLR, PLR, and LMR could be a predictor of the presence of EOC in all stage and subtypes of EOC.

In current study, using the cut-off value of CA125 level of 35UI/L, 11.7\% (88 of 841 EOC) of EOC were still missed. We found that high level of NLR and PLR with above optimal cutoff values increase the risk of endometrioid EOC, while low level of LMR were significantly associated with an increased risk of serous EOC in low CA125 level patients. This suggested that NLR, PLR, and LMR could be applied together with CA125 to predict the presence of EOC as well as the histological subtype of EOC in low CA125 level patients. It is reported that the frequency of elevated NLR $(N L R \geq 4)$ was highest in patients with serous EOC followed by clear cell, endometrioid, and mucinous types and the significant difference was observed between high and low NLR group in different histological types of EOC [37]. The diagnostic accuracy of inflammatory markers for distinguishing benign ovarian masses and malignant may be different according to histologic subtypes. However, this study lacked external validation of the main findings and further validation studies in an independent cohort are warranted. In addition, this was a retrospective study, a prospective study with large sample size would be needed to apply as a screening tool for ovarian cancer.

\section{Conclusions}

The findings of this study suggested that in addition to CA125 biomarkers, NLR, PLR and LMR could be used as predictors of EOC. We also found that preoperative inflammation markers may be a potential predictive marker of histology subtypes of EOC, which need to be validated by additional well-designed study.

\section{Supplementary Material}

Supplementary figures and tables. http://www.jcancer.org/v12p2687s1.pdf

\section{Acknowledgements}

This work was supported by the National Natural Science Foundation of China (81973113,
81320108022, 81470153, 81974439 and 81502877), Tianjin Science and Technology Committee Foundation (18YFZCSY00520, 16JCYBJC26600, and 16JCYBJC44000), the National Key Research and Development Program of China (2016YFC1302703).

\section{Competing Interests}

The authors have declared that no competing interest exists.

\section{References}

1. Khosravi-Shahi P, Cabezon-Gutierrez L. Antiangiogenic drugs in the treatment of advanced epithelial ovarian cancer. Anticancer Agents Med Chem. 2012; 12: 982-7.

2. Watrowski R, Heinze G, Jager C, Forster J, Zeillinger R. Usefulness of the preoperative platelet count in the diagnosis of adnexal tumors. Tumour Biol. 2016; 37: 12079-87.

3. Seckin KD, Karsli MF, Yucel B, Bestel M, Yildirim D, Canaz E, et al. The utility of tumor markers and neutrophil lymphocyte ratio in patients with an intraoperative diagnosis of mucinous borderline ovarian tumor. Eur J Obstet Gynecol Reprod Biol. 2016; 196: 60-3.

4. Lheureux S, Gourley C, Vergote I, Oza AM. Epithelial ovarian cancer. Lancet. 2019; 393: 1240-53.

5. Soletormos G, Duffy MJ, Othman Abu Hassan S, Verheijen RH, Tholander B, Bast RC Jr., et al. Clinical Use of Cancer Biomarkers in Epithelial Ovarian Cancer: Updated Guidelines From the European Group on Tumor Markers. Int J Gynecol Cancer. 2016; 26: 43-51.

6. Manegold-Brauer G, Bellin AK, Tercanli S, Lapaire O, Heinzelmann-Schwarz $\mathrm{V}$. The special role of ultrasound for screening, staging and surveillance of malignant ovarian tumors: distinction from other methods of diagnostic imaging. Arch Gynecol Obstet. 2014; 289: 491-8.

7. Moore RG, Miller MC, Disilvestro P, Landrum LM, Gajewski W, Ball JJ, et al. Evaluation of the diagnostic accuracy of the risk of ovarian malignancy algorithm in women with a pelvic mass. Obstet Gynecol. 2011; 118: 280-8.

8. Bristow RE, Smith A, Zhang Z, Chan DW, Crutcher G, Fung ET, et al. Ovarian malignancy risk stratification of the adnexal mass using a multivariate index assay. Gynecol Oncol. 2013; 128: 252-9.

9. Nossov V, Amneus M, Su F, Lang J, Janco JM, Reddy ST, et al. The early detection of ovarian cancer: from traditional methods to proteomics. Can we really do better than serum CA-125? Am J Obstet Gynecol. 2008; 199: 215-23.

10. Biggs WS, Marks ST. Diagnosis and Management of Adnexal Masses. Am Fam Physician. 2016; 93: 676-81.

11. Grivennikov SI, Greten FR, Karin M. Immunity, inflammation, and cancer. Cell. 2010; 140: 883-99.

12. Diem S, Schmid S, Krapf M, Flatz L, Born D, Jochum W, et al. Neutrophil-to-Lymphocyte ratio (NLR) and Platelet-to-Lymphocyte ratio (PLR) as prognostic markers in patients with non-small cell lung cancer (NSCLC) treated with nivolumab. Lung Cancer. 2017; 111: 176-81.

13. Ozmen S, Timur O, Calik I, Altinkaynak K, Simsek E, Gozcu H, et al. Neutrophil-lymphocyte ratio (NLR) and platelet-lymphocyte ratio (PLR) may be superior to $\mathrm{C}$-reactive protein (CRP) for predicting the occurrence of differentiated thyroid cancer. Endocr Regul. 2017; 51: 131-6.

14. He YF, Luo HQ, Wang W, Chen J, Yao YW, Yan Y, et al. Preoperative NLR and PLR in the middle or lower ESCC patients with radical operation. Eur J Cancer Care (Engl). 2017; 26.

15. Miyamoto R, Inagawa S, Sano N, Tadano S, Adachi S, Yamamoto M. The neutrophil-to-lymphocyte ratio (NLR) predicts short-term and long-term outcomes in gastric cancer patients. Eur J Surg Oncol. 2018; 44: 607-12.

16. $\mathrm{Hu} \mathrm{H}$, Yao X, Xie $\mathrm{X}, \mathrm{Wu} \mathrm{X}$, Zheng $\mathrm{C}$, Xia W, et al. Prognostic value of preoperative NLR, dNLR, PLR and CRP in surgical renal cell carcinoma patients. World J Urol. 2017; 35: 261-70.

17. Cho H, Hur HW, Kim SW, Kim SH, Kim JH, Kim YT, et al. Pre-treatment neutrophil to lymphocyte ratio is elevated in epithelial ovarian cancer and predicts survival after treatment. Cancer Immunol Immunother. 2009; 58: $15-23$

18. Yildirim M, Demir Cendek B, Filiz Avsar A. Differentiation between benign and malignant ovarian masses in the preoperative period using neutrophil-to-lymphocyte and platelet-to-lymphocyte ratios. Mol Clin Oncol. 2015; 3: 317-21.

19. Bakacak M, Serin S, Ercan O, Kostu B, Bostanci MS, Bakacak Z, et al. Utility of preoperative neutrophil-to-lymphocyte and platelet-to-lymphocyte ratios to distinguish malignant from benign ovarian masses. J Turk Ger Gynecol Assoc. 2016; 17: 21-5.

20. Eo WK, Kim KH, Park EJ, Kim HY, Kim HB, Koh SB, et al. Diagnostic accuracy of inflammatory markers for distinguishing malignant and benign ovarian masses. J Cancer. 2018; 9: 1165-72.

21. Bakacak M, Serin S, Ercan Ö, Köstü B, Bostancı MS, Bakacak Z, et al. Utility of preoperative neutrophil-to-lymphocyte and platelet-to-lymphocyte ratios to 
distinguish malignant from benign ovarian masses. J Turk Ger Gynecol Assoc. 2016; 17: 21-5.

22. Watrowski R, Zeillinger R. Simple laboratory score improves the preoperative diagnosis of adnexal mass. Tumour Biol. 2016; 37: 4343-9.

23. Wilailak S, Chan KK, Chen CA, Nam JH, Ochiai K, Aw TC, et al. Distinguishing benign from malignant pelvic mass utilizing an algorithm with HE4, menopausal status, and ultrasound findings. J Gynecol Oncol. 2015; 26: 46-53.

24. Topcu HO, Guzel AI, Ozer I, Kokanali MK, Gokturk U, Muftuoglu KH, et al. Comparison of neutrophil/lymphocyte and platelet/ lymphocyte ratios for predicting malignant potential of suspicious ovarian masses in gynecology practice. Asian Pac J Cancer Prev. 2014; 15: 6239-41.

25. Miyagi E, Maruyama Y, Mogami T, Numazaki R, Ikeda A, Yamamoto H, et al. Comparison of plasma amino acid profile-based index and CA125 in the diagnosis of epithelial ovarian cancers and borderline malignant tumors. Int J Clin Oncol. 2017; 22: 118-25.

26. Cymbaluk-Ploska A, Chudecka-Glaz A, Surowiec A, Pius-Sadowska E, Machalinski B, Menkiszak J. MMP3 in Comparison to CA 125, HE4 and the ROMA Algorithm in Differentiation of Ovarian Tumors. Asian Pac J Cancer Prev. 2016; 17: 2597-603.

27. Cho HY, Park SH, Park YH, Kim HB, Kang JB, et al. Comparison of HE4, CA125, and Risk of Ovarian Malignancy Algorithm in the Prediction of Ovarian Cancer in Korean Women. J Korean Med Sci. 2015; 30: 1777-83.

28. Chen S, Zhang L, Yan G, Cheng S, Fathy AH, Yan N, et al. Neutrophil-to-Lymphocyte Ratio Is a Potential Prognostic Biomarker in Patients with Ovarian Cancer: A Meta-Analysis. Biomed Res Int. 2017; 2017: 7943467.

29. Huang QT, Zhou L, Zeng WJ, Ma QQ, Wang W, Zhong M, et al. Prognostic Significance of Neutrophil-to-Lymphocyte Ratio in Ovarian Cancer: A Systematic Review and Meta-Analysis of Observational Studies. Cell Physiol Biochem. 2017; 41: 2411-8.

30. Polat M, Senol T, Ozkaya E, Ogurlu Pakay G, Cikman MS, Konukcu B, et al. Neutrophil to lymphocyte and platelet to lymphocyte ratios increase in ovarian tumors in the presence of frank stromal invasion. Clin Transl Oncol. 2016; 18: 457-63.

31. Eo WK, Chang HJ, Kwon SH, Koh SB, Kim YO, Ji YI, et al. The Lymphocyte-Monocyte Ratio Predicts Patient Survival and Aggressiveness of Ovarian Cancer. J Cancer. 2016; 7: 289-96.

32. Yildirim MA, Seckin KD, Togrul C, Baser E, Karsli MF, Gungor T, et al. Roles of neutrophil/lymphocyte and platelet/lymphocyte ratios in the early diagnosis of malignant ovarian masses. Asian Pac J Cancer Prev. 2014; 15: 6881-5.

33. Prodromidou A, Andreakos P, Kazakos C, Vlachos DE, Perrea D, Pergialiotis V. The diagnostic efficacy of platelet-to-lymphocyte ratio and neutrophil-to-lymphocyte ratio in ovarian cancer. Inflamm Res. 2017; 66: 467-75.

34. Raungkaewmanee S, Tangjitgamol S, Manusirivithaya S, Srijaipracharoen S, Thavaramara T. Platelet to lymphocyte ratio as a prognostic factor for epithelial ovarian cancer. J Gynecol Oncol. 2012; 23: 265-73.

35. Zhu JY, Liu CC, Wang L, Zhong M, Tang HL, Wang H. Peripheral blood lymphocyte-to-monocyte ratio as a prognostic factor in advanced epithelial ovarian cancer: a multicenter retrospective study. J Cancer. 2017; 8: 737-43.

36. Yang HM, Lou G. The relationship of preoperativelymphocyte-monocyte ratio and the clinicopathological characteristics and prognosis of patients with epithelial ovarian cancer. Zhonghua Zhong Liu Za Zhi. 2017; 39: 676-80.

37. Komura N, Mabuchi S, Yokoi E, Kozasa K, Kuroda H, Sasano T, et al. Comparison of clinical utility between neutrophil count and neutrophillymphocyte ratio in patients with ovarian cancer: a single institutional experience and a literature review. Int J Clin Oncol. 2018; 23: 104-13. 